\title{
Single-Jet Gas Cooling of In-Beam Foils or Specimens: Prediction of the Convective Heat-Transfer Coefficient
}

\author{
Gideon Steyn $^{1, \mathrm{a}), \mathrm{b})}$ and Christiaan Vermeulen ${ }^{2, \mathrm{c})}$ \\ ${ }^{1}$ iThemba LABS, P.O. Box 722, Somerset West, 7129, South Africa \\ ${ }^{2}$ Paul Scherrer Institute, Villigen-PSI, Switzerland \\ ${ }^{a)}$ Corresponding author: deon@tlabs.ac.za \\ b)URL: http://www.tlabs.ac.za \\ c) etienne.vermeulen@psi.ch
}

\begin{abstract}
An experiment was designed to study the effect of the jet direction on convective heat-transfer coefficients in single-jet gas cooling of a small heated surface, such as typically induced by an accelerated ion beam on a thin foil or specimen. The hot spot was provided using a small electrically heated plate. Heat-transfer calculations were performed using simple empirical methods based on dimensional analysis as well as by means of an advanced computational fluid dynamics (CFD) code. The results provide an explanation for the observed turbulent cooling of a double-foil, Havar beam window with fast-flowing helium, located on a target station for radionuclide production with a $66 \mathrm{MeV}$ proton beam at a cyclotron facility.
\end{abstract}

\section{INTRODUCTION}

Various applications utilize an impinging stream of gas for the cooling of metal foils and/or other material specimens while under bombardment in an accelerated particle beam. Helium is often used for this purpose (see e.g. [1-4] as typical examples) but other gasses are also sometimes employed. Noble gasses can provide cooling as well as an inert environment for the protection of the foils/specimens.

At iThemba LABS, radionuclide production targets are bombarded outside the cyclotron and beamline vacuum. In addition to preserving the integrity of the vacuum, the ability to rapidly transfer targets to and from a target station is greatly simplified by eliminating the need to repeatedly break and restore the vacuum. The beamlines to the target stations have been provided with beam exit windows consisting of two closely-spaced thin metal foils (Havar) cooled by helium flowing between them. These gas-cooled, double-foil windows are thin enough to cause minimal energy degradation to the beam but provide a strong enough barrier to maintain the vacuum behind it for extended periods of time. They do have a finite life-time due to radiation damage, nevertheless, most window assemblies last many months and some even several years. A schematic diagram of a window and target assembly is shown in Fig. 1.

A significant number of papers dealing with the gas cooling of thin foils or specimens in ion-beam applications have been published over the years. Several authors estimated the convective heat-transfer coefficient by considering the in-beam surface to be equivalent to a small heated surface on the inner wall of a circular tube or duct through which the coolant flows. It is not clear when this practice started but the oldest paper in our possession that follows this approach is by Liljenzin [5], who used the Dittus-Boelter equation [6] to estimate the convective heat-transfer coefficient. This equation was derived using the methods of dimensional analysis and correlations with experimental data. The Dittus-Boelter equation is the oldest of several similar approaches to forced convection heat transfer in tubes or ducts. Later refinements, such as the Sieder-Tate and Petukhov-Kirillov equations, are still widely employed today.

During the development of the first bombardment station for radionuclide production at iThemba LABS, an experiment was performed to measure convective heat-transfer coefficients for helium flowing in a prototype doublefoil window assembly. An experimental assembly was built with the exact dimensions and lay-out of a real beamwindow assembly except that the hot beam spots were simulated with electrically heated elements. That work reported significantly higher convective heat-transfer coefficients than predictions based on the Dittus-Boelter, Sieder-Tate and Petukhov-Kirillov equations [7]. The large discrepancy was finally understood to be related to the neglect of thermal 


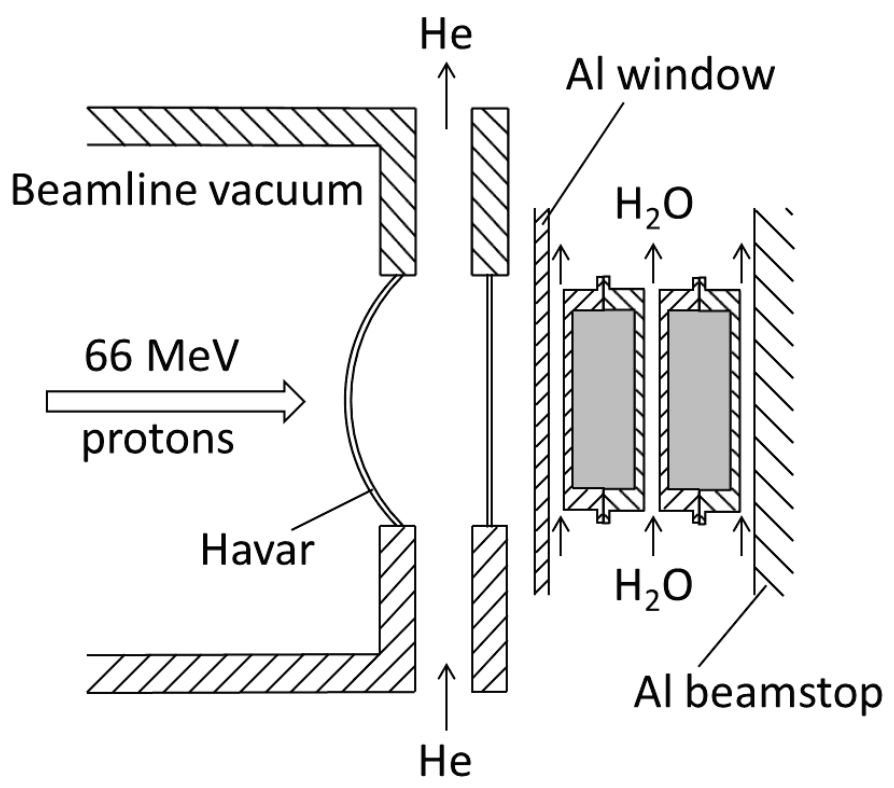

FIGURE 1. Schematic diagram of two water-cooled, encapsulated targets in a tandem geometry, located behind a helium-cooled, double-foil Havar window assembly.

entrance effects. The above-mentioned equations are valid for long heated pipes (i.e. for a length much longer than the inner diameter of the flow passage) where both the hydrodynamic and thermal boundary layers become fully developed. Even if the hydrodynamic boundary layer becomes fully developed but not the thermal boundary layer, a significant enhancement of the convection heat transfer can be expected. Such effects are seen in the entrance regions of heated tubes, through which a coolant is flowing. In fact, the enhancement is most notable in the region where the length-to-diameter ratio is smaller than unity (i.e. $x / D<1$ ). It was argued in Ref. [8] that the small heated areas made by focused beams on window foils cannot merely be treated as hot spots on the inner walls of tubes unless these spots are located in the entrance region of the tube. Predictions of convective heat-transfer coefficients in the thermal entry region can be made using a formalism by Kays and Perkins [9], amongst others. The measured and predicted convective heat-transfer coefficients using the duct equations are presented in Table 1. Also shown in the table is the length-to-diameter ratio at which a prediction using the formalism by Kays and Perkins gives the same result as the experimentally measured value $\left(h=0.244 \mathrm{~W} \mathrm{~cm}^{-2}{ }^{\circ} \mathrm{C}^{-1}\right.$ only when $\left.x / D=0.42\right)$. This result is interesting as it clearly illustrates the enhancement of the convective heat-transfer coefficient in the thermal entrance region. On the other hand, the Kays and Perkins formalism (or similar approaches) does not provide a method to make quantitative predictions due to additional parameters that are inter alia unknown.

Recently, calculations based on a prescription by Chang et al. [10] for single-jet impingement heat transfer showed much promise. This topic featured at the $13^{\text {th }}$ International Workshop on Targetry and Target Chemistry (WTTC 13) which was held in Roskilde, Denmark, in 2010. During that workshop, work by Gagnon et al. [11] on the modelling of a water-cooled solid cyclotron target using finite element analysis was presented. These authors used the Dittus-Boelter, Sieder-Tate and the formalism by Chang et al. to calculate convective heat-transfer coefficients for use in their model. They also performed measurements of the target temperature at the water/solid boundary for various values of the power deposited by the beam. An important aspect of the target design is that the cooling water enters the target assembly from the back through a single central inlet port and the flow direction of the jet is perpendicular to the heated surface, parallel but opposite to the beam direction. After impact with the hot surface, the water flows radially outwards towards two outlet ports. It was found that the Dittus-Boelter and Sieder-Tate equations underestimated the convective heat-transfer coefficient considerably, very similar to what we observed for the helium cooling of beam windows. In sharp contrast, the calculations using the formalism of Chang et al. gave results in excellent agreement with the experimental measurements. In the original paper by Chang, the coolant is freon and the direction of flow is perpendicular to the heated surface. It is interesting that a formulation derived from correlations using liquid freon 
TABLE 1. Convective heat-transfer coefficients relevant to the helium-cooled beam-window assemblies of the horizontal-beam target stations at iThemba LABS.

\begin{tabular}{lcc}
\hline Method & $\begin{array}{c}h \\
\left(\mathrm{~W} \mathrm{~cm}{ }^{-2}{ }^{\circ} \mathrm{C}^{-1}\right)\end{array}$ & $x / D$ \\
\hline Measured [7] & 0.244 & \\
Dittus-Boelter [7] & 0.086 & \\
Sieder-Tate [7] & 0.094 & \\
Petukhov-Kirillov [7] & 0.078 & \\
Kays and Perkins [9] & 0.244 & 0.42 \\
Chang et al. (single jet) [10] & 0.228 & \\
\hline
\end{tabular}

Helium pressure: 1.25 bar.

Bulk flow rate: $125 \mathrm{~m}^{3} / \mathrm{h}$.

Helium inlet temperature: $300 \mathrm{~K}$ (room temperature).

as coolant gives good quantitative results also for forced convection with water as coolant. What about the predictive power for the case of helium as the coolant in a typical double-foil window assembly?

The final value in Table 1 is our calculation using Chang's formalism for a single helium jet. The jet diameter was taken as the hydraulic diameter of the entrance port, which is in close proximity to the window foils. This prediction is within 7\% of the measured value, in sharp contrast to the values from the Dittus-Boelter, Sieder-Tate and PetukhovKirillov equations which underpredict the convective heat-transfer coefficient by about a factor of 3 .

Chang's approach for single-jet impingement heat transfer has been applied to the helium-cooled window assembly in a rather "cold-blooded" fashion, in that the direction of flow in the window assembly is not at all perpendicular to the heated surface. In fact, the flow in the window assembly is horizontal to the surface! This, admittedly, is not something that can be ignored. In order to investigate the dependence on the jet configuration, i.e. the direction of flow onto the heated surface, an experiment was designed in which the incident angle of the jet could be adjusted between $0^{\circ}$ and $90^{\circ}$. New measurements of convective heat-transfer coefficients are presented, employing an electrically heated element to simulate the beam. In order to simplify the experimental set-up, air was used as the coolant, which will be discussed later. We believe this is justified as results obtained from one particular gas can readily be scaled to other gasses. The new measurements are compared with predictions by means of the duct equations as well as Chang's formalism. Modelling of the experimental set-up with a commercial Computational Fluid Dynamics (CFD) code has also been performed. Results obtained from two different CFD prescriptions are compared, namely the K-epsilon turbulence model and the shear stress transport (SST) K-omega model [12-14].

In the following sections, details of the formalism by Chang et al. as well as some comments on the underlying dimensional analysis will be discussed first. This will be followed by a description of the experimental set-up, measured results and calculations based on the methods of dimensional analysis. A summary of the results from the advanced modelling using a CFD code will then be presented, followed by some comments in conclusion.

\section{CHANG'S FORMALISM FOR SINGLE-JET IMPINGEMENT HEAT TRANSFER}

Chang et al. [10] employed jets with circular orifices. As is often done, we substituted the diameter in the equations with the hydraulic diameter in cases where the orifice had a shape different from circular. The following expression gives the Nusselt number at the point of impact (the so-called stagnation point):

$$
\mathrm{Nu}(0)=0.660 \operatorname{Re}_{j}^{0.574} \operatorname{Pr}^{0.4}\left(z / d_{j}\right)^{-0.106}
$$

where $\operatorname{Re}_{j}$ is the Reynolds number evaluated with the characteristic length taken as the exit nozzle diameter of the jet, $\operatorname{Pr}$ is the Prandtl number, $z$ is the distance from the nozzle exit to the stagnation point, and $d_{j}$ is the nozzle exit diameter. The dimensionless groups for the single-jet case are as follows:

$$
\mathrm{Nu}=\frac{h d_{j}}{k} ; \operatorname{Re}=\frac{\rho v d_{j}}{\mu} ; \operatorname{Pr}=\frac{c_{p} \mu}{k} ;
$$


where $h$ is the convective heat-transfer coefficient, $k$ is the thermal conductivity of the coolant, $\rho$ is the density (or specific gravity) of the coolant, $v$ is the bulk velocity of the coolant where the jet exits the nozzle, $\mu$ is the dynamic viscosity of the coolant, and $c_{p}$ is the specific heat of the coolant. The Nusselt number is often described as a dimensionless form of the convective heat-transfer coefficient. Once the Nusselt number is known, $h$ can be calculated. In single-jet impingement heat transfer, there is a radial decrease in the value of the Nusselt number away from the stagnation point. A local average Nusselt number for a region within a radius $r$ from the stagnation point is given by

$$
\begin{array}{ll}
\frac{\overline{\mathrm{Nu}}(r)}{\mathrm{Nu}(0)}=\left[1+0.1147\left(r / d_{j}\right)^{1.81}\right]^{-1} & \text { for } r / d_{j} \leq 1.25, \\
\frac{\overline{\mathrm{Nu}}(r)}{\mathrm{Nu}(0)}=1.0632\left(r / d_{j}\right)^{-0.62} & \text { for } r / d_{j}>1.25 .
\end{array}
$$

The local average convective heat-transfer coefficient is then given by

$$
\bar{h}(r)=\frac{\overline{\mathrm{Nu}}(r) k}{d_{j}} .
$$

The expressions of the well-known duct equations, which have functional forms with some resemblance to Eq. 1 but different coefficients, will not be presented here in detail - they can be found in many handbooks on heat transfer (e.g. Holman [15]) as well as in a previous paper [8] and references therein. They are based on the relation

$$
\mathrm{Nu}=C \operatorname{Re}^{m} \operatorname{Pr}^{n},
$$

where $\mathrm{Nu}, \mathrm{Re}$ and $\mathrm{Pr}$ are dimensionless groups (as in Eq. 2 but with the nozzle exit diameter replaced by the hydraulic diameter of the duct); $C, m$ and $n$ are coefficients (real numbers) to be determined experimentally by correlations with measured data. In addition, the Sieder-Tate equation includes a correction for the difference in viscosity at the heated duct wall and the bulk temperature of the coolant, which may be substantial. Petukhov and Kirillov added a friction factor to explicitly take into account the drag caused by the surface roughness of the duct on the coolant medium.

\section{NEW EXPERIMENTAL MEASUREMENTS}

\section{Experimental Set-Up for Single Jets}

An experiment was designed to measure convective heat-transfer coefficients for a gas incident onto a small heated surface, similar in size to a typical beam spot in the vertical beam target station (VBTS) at iThemba LABS [16]. The main aim was to investigate the effect of changing the angle of incidence of the jet onto the heated surface while keeping everything else the same. For the purposes of the experiment, it was not considered essential that the gas had to be helium circulating in a closed loop. In order to simplify the experimental set-up, it was decided to use air at ambient conditions as the coolant. This eliminated the need for a gas-tight, closed-loop system and a heat exchanger. A schematic diagram of the system is shown in Fig. 2 with further details in Fig. 3.

The main aspects of the experimental set-up are as follows: A Northey twin-rotor compressor supplies a bulk flow of up to $125 \mathrm{~m}^{3} / \mathrm{h}$ to a $25 \mathrm{~L}$ buffer tank. Tubing with a $40 \mathrm{~mm}$ inner diameter connects the tank to a heated plate assembly. A commercial electric element (manufactured by Swift Heat \& Control: $230 \mathrm{~V}$ AC, $400 \mathrm{~W}$ ) is embedded into the aluminium plate, as shown in Fig. 3. The aluminium plate assembly is thermally insulated with mineral wool (supplied by Rockwool International, Denmark) except at the circular gas-cooled surface. The heated surface $(A)$ has a diameter of $36 \mathrm{~mm}$. A k-type thermocouple is used to measure the temperature $(T)$ at the solid/gas interface. The $\mathrm{AC}$ voltage can be adjusted with a Variac and Fluke instrumentation is used to read out the voltage and current values. All temperature, voltage and current values can also be streamed to a computer in 1 second intervals. A small chamber with a hemispherical shape (or dome) fits over the heated surface. In fact, five of these chambers were constructed with different jet angles but otherwise they are identical. Measurements were performed at jet angles of $0^{\circ}, 22.5^{\circ}$, $45^{\circ}, 67.5^{\circ}$ and $90^{\circ}$. The surface area adjacent to the heated surface is made of Tufnol, a material with more than two orders of magnitude lower thermal conductivity than aluminium. Also, the Tufnol plate was machined so that the material in contact with the aluminium plate was only $1 \mathrm{~mm}$ thick. This provided a single hot surface within a region predominantly at room temperature. Several rectangular apertures along the bottom edge of the dome serve as exit 


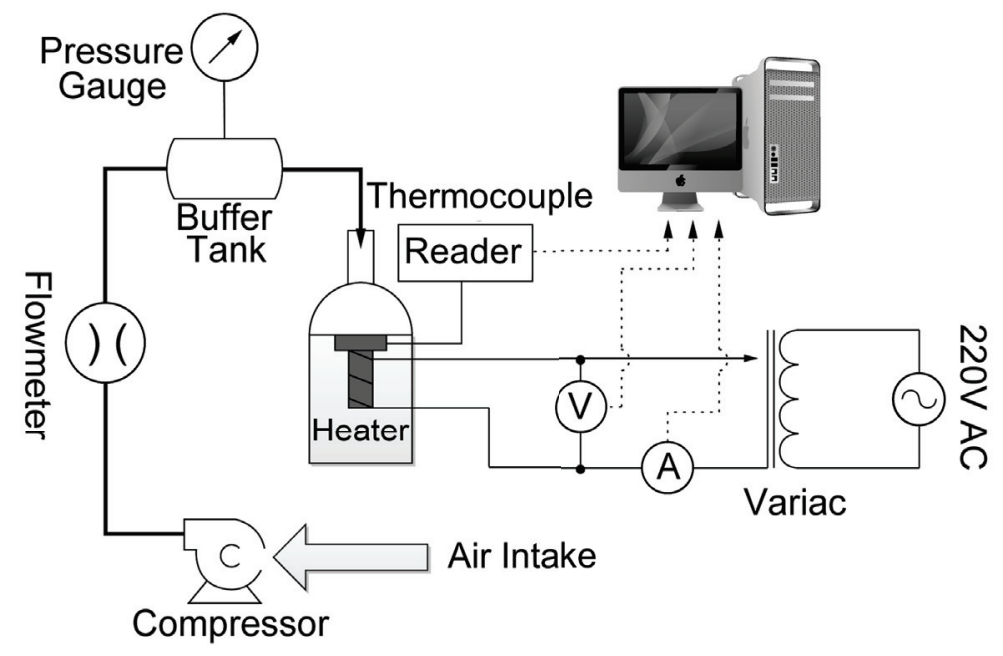

FIGURE 2. Schematic diagram of the experimental set-up to measure convective heat-transfer coefficients for air flowing onto a small heated surface.
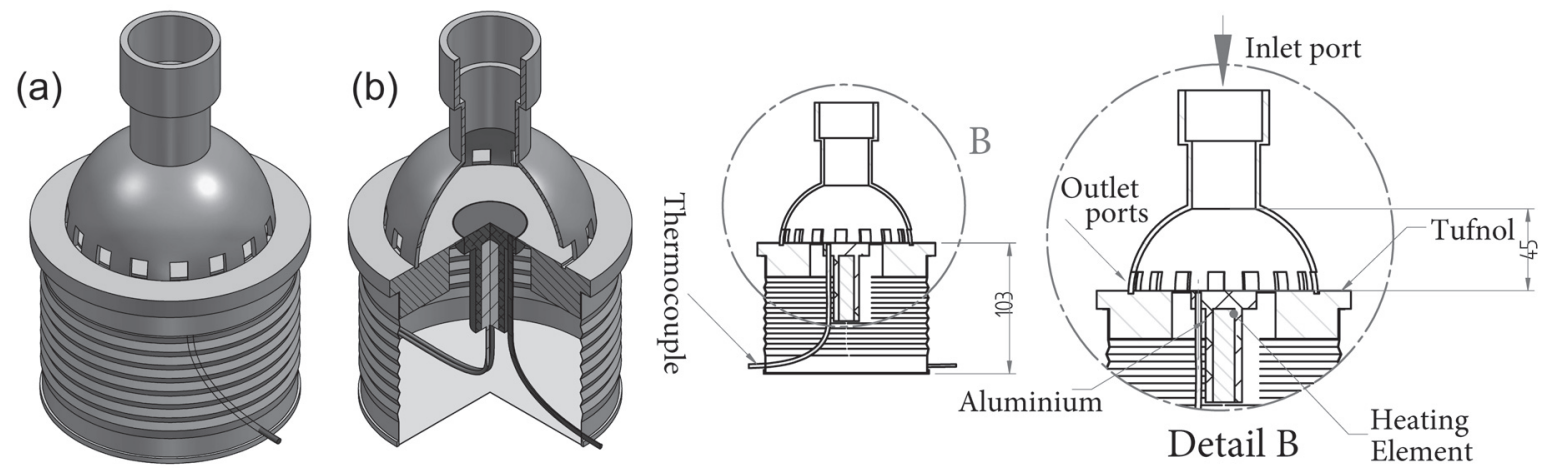

FIGURE 3. LEFT: (a) 3D view of the hemispherical chamber (or dome) and electrically heated plate assembly. (b) The same view but with a cut-away of the assembly to show the heated surface and single jet. RIGHT: Cross-sectional views of the electrically heated plate assembly for measuring convective heat-transfer coefficients with a single jet. Note the single inlet port at the top and several rectangular outlet ports along the bottom edge of the hemispherical chamber.

ports for the gas (nine in total, each $10 \mathrm{~mm}$ wide and $11 \mathrm{~mm}$ high on one side of the dome only, opposite to the inlet side). The differential pressure between the buffer tank and outside atmosphere never exceeded 15 mbar, thus, for all practical purposes the pressure of the gas inside the dome was considered to be at a constant value of 1 bar.

The thermal losses due to mechanisms other than forced convection were determined by covering the heated surface with insulating wool and measuring the electrical power $(q)$ versus temperature characteristics of the system for steady-state conditions without flow. These values were subtracted from the corresponding values with flow. Cooling power densities (i.e. the heat-transfer rates) were measured at surface temperatures of nominally 100, 150, 200, 250 and $300^{\circ} \mathrm{C}$.

\section{Data Analysis}

When the values of the power density $(q / A)$ are plotted versus the difference in temperature between the surface and the coolant $\left(T-T_{0}\right)$, the convective heat-transfer coefficient is given by the slope of a straight line fitted through the measured data points. A representative example is shown in Fig. 4 . In this case the jet direction was $0^{\circ}$, i.e. parallel to the heated surface. Note that the adiabatic heating of the gas was determined to be about $14{ }^{\circ} \mathrm{C}$ by running the system with the electric element switched off, which raised the coolant temperature somewhat above the room 


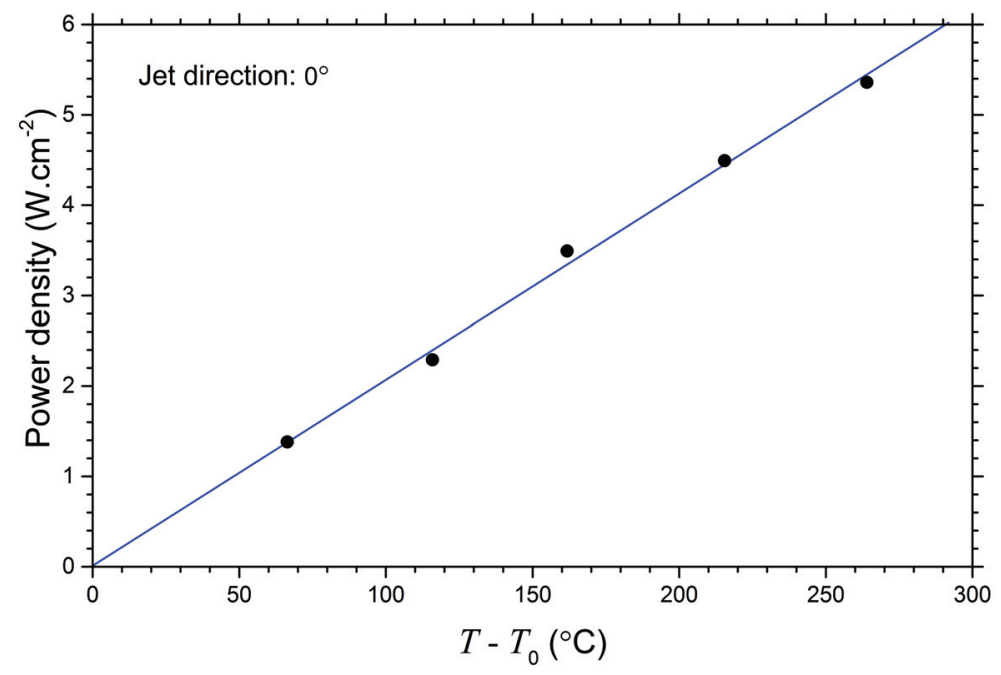

FIGURE 4. Measured power density $(q / A)$ versus the difference in temperature between the surface and the coolant $\left(T-T_{0}\right)$ in the case of flow parallel to the heated surface. The coolant was air at a pressure of 1 bar and a flow rate of $125 \mathrm{~m}^{3} / \mathrm{h}$. The diameter of the single jet was $36 \mathrm{~mm}$.

temperature. The uncertainty associated with each $h$-value determined from the fitting procedure was taken as one standard deviation of the $h$-values determined from the individual measurements at discrete temperatures.

\section{Results and discussion - single jets}

The measured convective heat-transfer coefficients are shown in Fig. 5 as a function of the jet angle. Also shown in Fig. 5 are calculated values according to the Chang, Dittus-Boelter, Sieder-Tate and Petukhov-Kirillov equations. Note that these calculated values are shown as horizontal lines in the figure for purposes of comparison with the measured values, however, they are not to be understood as functions of the jet angle. The two short-dashed lines are calculations using the Chang equations for flow rates adjusted by $-6 \%$ and $+6 \%$ relative to the nominal value of $125 \mathrm{~m}^{3} / \mathrm{h}$, respectively, as an indication of the measure of uncertainty described above. The small schematic diagrams of the dome at the top of the figure indicate the flow directions. The bulk velocity of the jet was nominally $36 \mathrm{~m} / \mathrm{s}$.

This result is rather remarkable. There is very little difference between the measured heat-transfer coefficients except at a jet angle of $45^{\circ}$, where the value is slightly lower. The angular dependence is indeed weak. We believe that this result points significantly towards an explanation why the formalism of Chang et al., established for a jet direction of $90^{\circ}$, yields a surprisingly good value also at $0^{\circ}$ in a helium-cooled window assembly (see Table 1). At least, we have evidence of a case where a change in jet direction from $90^{\circ}$ to $0^{\circ}$ made little difference to the convection cooling. Prior to the experiment, it was expected (or rather hoped) to find only a weak dependence on jet angle, perhaps a plateau with a slow drop-off towards smaller angles. Instead, we see the largest difference at a jet angle of $45^{\circ}$, which is rather intriguing. Repeated measurements consistently reproduced this minimum at $45^{\circ}$. It is interesting to compare some of the convective heat-transfer values at $90^{\circ}$ : Measured $\left(h=0.0212 \mathrm{~W} \mathrm{~cm}^{-2}{ }^{\circ} \mathrm{C}^{-1}\right)$; Chang $(h=$ $\left.0.0258 \mathrm{~W} \mathrm{~cm}^{-2}{ }^{\circ} \mathrm{C}^{-1}\right)$; Dittus-Boelter $\left(h=0.0125 \mathrm{~W} \mathrm{~cm}^{-2}{ }^{\circ} \mathrm{C}^{-1}\right)$. Chang's formalism gives a $21.7 \%$ overprediction relative to the measured value. The Dittus-Boelter equation, on the other hand, gives a $69.6 \%$ underprediction. Thus the Chang result is significantly closer (perhaps somewhat obscured in Fig. 5).

The experimental set-up was found to be very stable. Individual measurements repeated on different days yielded the same results. It seems that if a small heated surface is enclosed inside a compact chamber through which a coolant gas flows at a large flow rate, such that the time for an exchange of the gas from the chamber is small compared to the relaxation time of the system, the exact orientation of the heated surface is of secondary importance. With "relaxation time" we mean the characteristic time to respond to a change of conditions or to recover from a perturbation to the system. With "small" we mean dimensions not significantly larger than that of the jet diameter. This statement is made reservedly - it will be changed or rephrased if better insight warrants a better description. 


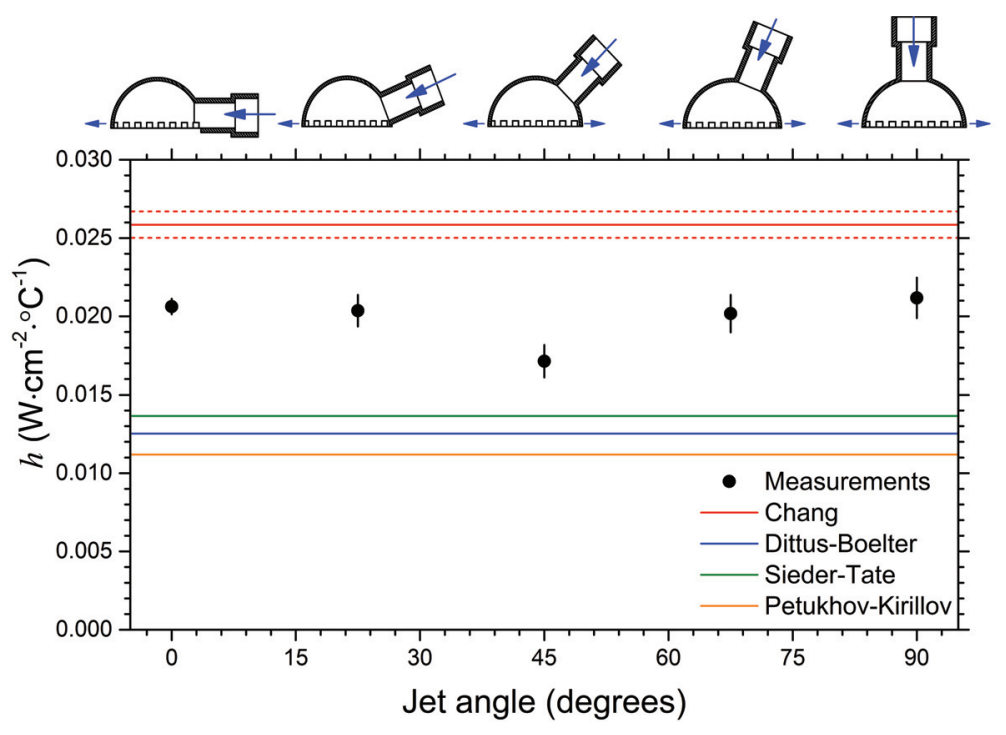

FIGURE 5. Measured convective heat-transfer coefficients versus jet angle. The bulk linear velocity of the air was nominally $36 \mathrm{~m} / \mathrm{s}$. Some calculated values are also shown, as indicated. See also caption to Fig. 4 for further details.

\section{Simple modelling using the method of finite differences}

As already mentioned, the heated surface was chosen to have an area similar to the expected beam spot on a VBTS target and beam-window assembly. In addition, the heating element was positioned such that the contact area onto the aluminium plate approximately modelled the annular surface of a circularly swept beam. Temperature profile calculations were performed using the method of finite differences $[15,16]$. A boundary condition was specified that no conduction should occur between the radial edge of the plate and surrounding materials. Heating was from below onto one flat surface and convection cooling was applied on the other flat surface of the heated plate. Relaxation was first performed in the axial direction by allowing conduction in only that direction until a steady-state was obtained. Relaxation was then performed in the radial direction until a steady state was again obtained. The method followed was to adjust the thermal load until the edge temperature of the plate reached a particular value at which a measurement was performed. The power required to achieve this could then be directly compared with the measured electrical power supplied to the heated plate. Generally, excellent agreement was found.

The thickness of the heated plate in the experiment was chosen to be $10 \mathrm{~mm}$, which is rather thick. This was done for a reason, namely, to keep the entire heated surface onto which the convection cooling was applied constant, for all practical purposes. Figure 6 shows the calculated radial temperature profile of the heated surface for this case, where the edge temperature was set at $300^{\circ} \mathrm{C}$. The convective heat-transfer coefficient measured at $0^{\circ}$ was adopted, namely $h=0.0206 \mathrm{~W} \mathrm{~cm}{ }^{-2}{ }^{\circ} \mathrm{C}^{-1}$. The power required to reach a steady-state with $300{ }^{\circ} \mathrm{C}$ edge temperature was $55.8 \mathrm{~W}$, in good agreement with the corresponding experimental value of $54.5 \mathrm{~W}$ (in which case $300{ }^{\circ} \mathrm{C}$ was measured at the edge). This is very reassuring. As can be seen in the figure, the variation in temperature across the heated surface is less than $2{ }^{\circ} \mathrm{C}$ in this case.

Also shown in Fig. 6 are radial temperature profiles of the heated surface should the plate be made thinner, as indicated. For these calculations, the thermal load was kept unchanged at a value of $55.8 \mathrm{~W}$. It is clear that the radial temperature variation increases significantly with decreasing plate thickness. With a plate thickness of $1 \mathrm{~mm}$, the radial temperature variation is slightly more than $17^{\circ} \mathrm{C}$, which would no longer be negligible. This behavior is to be expected as the cross-sectional area through which the heat has to spread radially by conduction is smaller when the plate becomes thinner. A thin window would have been advantageous from the point of view of the response time of the system, i.e. a steady state would be arrived at sooner after an adjustment to the thermal load. The thicker plate used in the actual experiment made the response somewhat tardy but this was not a disadvantage of any real significance. 


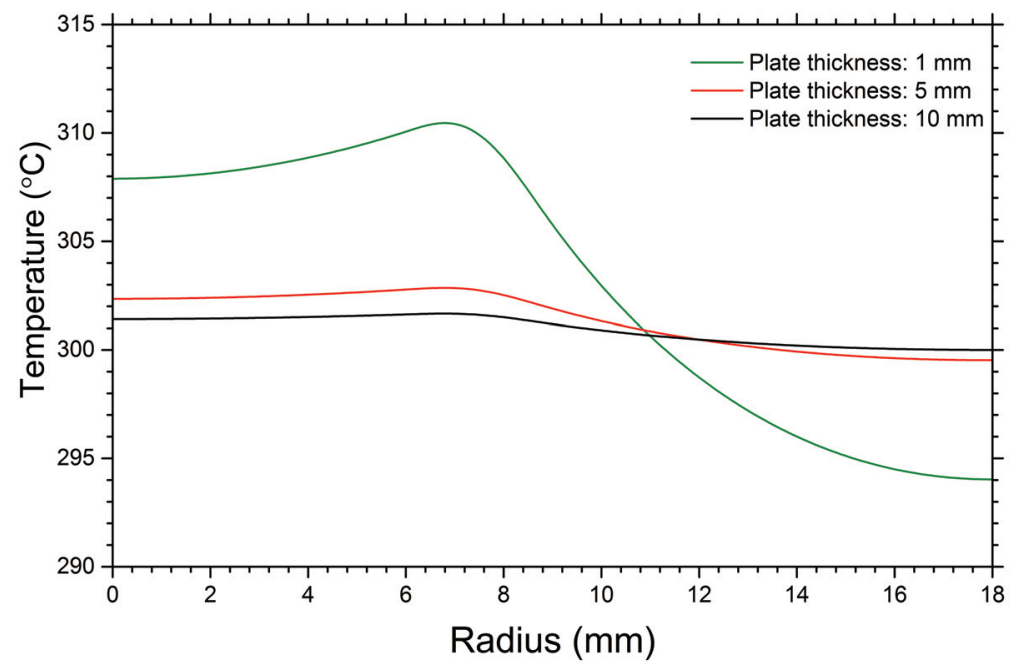

FIGURE 6. Calculated steady-state radial temperature profiles of the heated plate for different plate thicknesses. A convective heat-transfer coefficient of $h=0.0206 \mathrm{~W} \mathrm{~cm}^{-2}{ }^{\circ} \mathrm{C}^{-1}$ and a thermal load of $55.8 \mathrm{~W}$ (see text) were specified.

\section{ADVANCED MODELLING USING CFD}

From the outset of this project, it was planned to investigate advanced modelling using modern codes in which Computational Fluid Dynamics (CFD) and Finite Element Analysis (FEA) techniques for thermal analysis have been implemented. Such work, however, was to follow initial work using simpler techniques based on the finite difference technique and dimensional analysis. Both approaches are believed to be useful and important.

The experimental set-up for single-jet cooling was modelled using the commercial code Autodesk Simulation CFD 2014 (formerly called CFDesign). The geometry of the set-up, as designed with the drafting code Solid Edge, was imported via AutoCAD Inventor into the Simulation CFD code and further refined by specifying the various materials, surfaces and flow passages. In this way, models of the geometries with flow at $0^{\circ}, 45^{\circ}$ and $90^{\circ}$ relative to the heated surface were established. It was possible to arrive at the first calculated results relatively quickly as the Simulation CFD code has many default options. It is also a reasonably user friendly code with a well-developed user interface to provide the necessary input, such as boundary conditions, mesh sizes and model choices. The coolant was specified as air with a temperature of $25^{\circ} \mathrm{C}$ and a bulk flow rate of $125 \mathrm{~m}^{3} / \mathrm{h}$, similar to the actual experiment. A constant heat flux of $q=5.4 \mathrm{~W} \mathrm{~cm}^{-2}$ was specified for the heated surface, which is equivalent to a total thermal load of $55 \mathrm{~W}$. The first calculations were performed mostly using "automatic" selections, allowing the program to determine a mesh size by its own internal algorithms. The user is allowed to alter the mesh size by means of a slider. Since some sensitivity to the choice of mesh size was evident, it was decided to reduce the mesh but not to exceed a total of $10^{6}$ elements with the K-epsilon turbulence model (see below). Figure 7 shows an example of a velocity magnitude profile for the $45^{\circ}$ jet scenario. Such a run created just over 743000 elements and typically took several hours to complete on a single high-end PC.

The Autodesk Simulation CFD 2014 code has eight built-in turbulence models. The default is the K-epsilon model, which is described as one of the most common turbulence models of CFD. The online literature of the code gives a fairly comprehensive description of each of these models. By discounting those models intended for low Reynolds numbers, transition flow or otherwise not recommended for our type of internal-flow problem, only four turbulence models remain. Only two of these have been investigated at this time, namely the default K-epsilon model and the SST K-omega model. A short description on each is given below.

\section{The K-epsilon turbulence model}

This is a general purpose model that is reported to perform well across a large number of applications. The first variable, $K$, determines the kinetic energy in the turbulence and the second variable, $\epsilon$, is the so-called "turbulent 


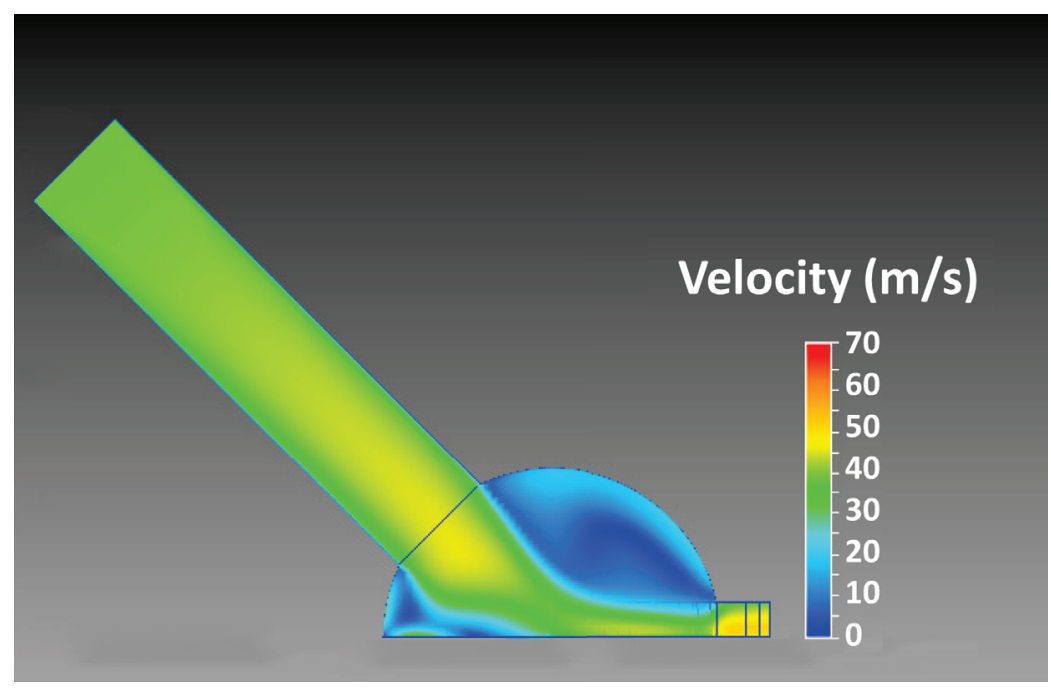

FIGURE 7. A velocity profile for a $45^{\circ}$ impingement of the coolant in the experimental set-up as modelled with the code Autodesk Simulation CFD 2014. Note that this is the XY median plane, showing the magnitude of the maximum components of the flow vectors in that plane.

dissipation", which describes the scale of the turbulence [12]. According to Bardina et al. [13], the K-epsilon model gives good results for wall-bounded and internal flows only in cases where the mean pressure gradients are small. These authors also report that the K-epsilon model would be an inappropriate choice for modelling heat transfer near flow inlets. As already mentioned, the small heated surface of a beam spot in a gas-cooled window assembly can be thought of as a thermal entry-region problem, thus, it is possible that the K-epsilon model may not be the best choice in this particular case. It has been reported that under highly turbulent conditions (i.e. high Reynolds numbers), the $\mathrm{K}$-epsilon model overpredicts the mixing inside the wall boundary layer [12], therefore overpredicting the convection heat transfer. A so-called "damping function" or "wall function" is employed to describe the strong attenuation of the normal component of turbulence in the vicinity of the wall. Several such damping functions exist and we could not with certainty determine which is implemented in the code, but it is probably the so-called "standard wall function" (SWF) in the case of the default calculation.

The maximum number of iterations was set to 2000 but convergence was typically reached after 700-800 iterations in all the runs performed. A run typically lasted between 5 and 6 hours. Except for adjusting the mesh size to the minimum value allowed, no further parameter adjustments were made. We want to stress this point - no fine tuning of any description was attempted by making parameter adjustments. Results for three of the impingement angles are shown in Table 2. It is evident that the convective heat-transfer coefficients obtained from the CFD calculations are somewhat higher than the corresponding experimental values. Interestingly, the results for the $0^{\circ}$ and $45^{\circ}$ jet angles are nearly the same, with some enhancement in the convection heat transfer predicted at $90^{\circ}$.

\section{The SST K-omega turbulence model}

The shear stress transport (SST) K-omega model is described as an eddy-viscosity model which has become very popular in recent years. Its behavior is the same as the K-epsilon model in free stream regions of the flow but the treatment is very different near wall boundaries. It does not use any ad-hoc damping functions to describe the viscous sub-layer flow near a wall. Instead, it simulates turbulence all the way to the wall boundary but requires a very fine mesh in the boundary layer region. With this choice, one can specify up to ten extra mesh layers in the boundary layer region using the "Mesh Enhancement dialog" of the code. Indeed, in our calculations we selected a value of 10 for this parameter (the default value is 3 ). The price to pay is that any calculation becomes much more resource intensive and requires a significantly longer execution time.

The results are shown in Table 3. For the $0^{\circ}$-jet case, the agreement between the measured convective heat-transfer coefficient and the corresponding CFD result is almost perfect. At $90^{\circ}$, the CFD result is about $27 \%$ higher than the measured value. Interestingly, the CFD result at $90^{\circ}$ is in excellent agreement with the prediction by means of the 
Chang formalism (see Fig. 5) - the difference being less than 5\%. As can be seen from Table 3, the predicted convective heat-transfer coefficients obtained by means of the CFD calculations also do not display a strong dependence on the jet angle, confirming the experimental observations. While the curious experimental minimum at $45^{\circ}$ was not reproduced in the CFD calculations, the overall agreement between the experimental and CFD results is satisfactory. This is very reassuring. The SST K-omega turbulence model is clearly a better choice than the K-epsilon turbulence model in this regime of heat transfer. From comments by other authors (e.g. Pattamatta et al. [14]), this is not unexpected.

TABLE 2. Results of Autodesk Simulation CFD calculations for different impingement angles using the K-epsilon turbulence model.

\begin{tabular}{cccccc}
\hline Jet angle & $\begin{array}{c}T_{\min } \\
{ }^{\circ} \mathrm{C}\end{array}$ & $\begin{array}{c}T_{\max } \\
{ }^{\circ} \mathrm{C}\end{array}$ & $\begin{array}{c}\bar{T} \\
{ }^{\circ} \mathrm{C}\end{array}$ & $\begin{array}{c}h \text { [CFD] } \\
\left(\mathrm{W} \mathrm{cm}^{-2}{ }^{\circ} \mathrm{C}^{-1}\right)\end{array}$ & $\begin{array}{c}h \text { [measured] } \\
\left(\mathrm{W} \mathrm{cm}^{-2}{ }^{\circ} \mathrm{C}^{-1}\right)\end{array}$ \\
\hline $0^{\circ}$ & 207.92 & 213.56 & 211.72 & 0.0289 & $0.0206 \pm 0.0005$ \\
$45^{\circ}$ & 207.39 & 212.94 & 210.96 & 0.0290 & $0.0171 \pm 0.0010$ \\
$90^{\circ}$ & 165.29 & 168.98 & 167.21 & 0.0380 & $0.0212 \pm 0.0013$ \\
\hline
\end{tabular}

TABLE 3. Results of Autodesk Simulation CFD calculations for different impingement angles using the SST K-omega turbulence model.

\begin{tabular}{cccccc}
\hline Jet angle & $\begin{array}{c}T_{\min } \\
{ }^{\circ} \mathrm{C}\end{array}$ & $\begin{array}{c}T_{\max } \\
{ }^{\circ} \mathrm{C}\end{array}$ & $\begin{array}{c}\bar{T} \\
{ }^{\circ} \mathrm{C}\end{array}$ & $\begin{array}{c}h \text { [CFD] } \\
\left(\mathrm{W} \mathrm{cm}^{-2}{ }^{\circ} \mathrm{C}^{-1}\right)\end{array}$ & $\begin{array}{c}h \text { [measured] } \\
\left(\mathrm{W} \mathrm{cm}^{-2}{ }^{\circ} \mathrm{C}^{-1}\right)\end{array}$ \\
\hline $0^{\circ}$ & 273.43 & 283.87 & 281.01 & 0.0211 & $0.0206 \pm 0.0005$ \\
$45^{\circ}$ & 265.05 & 269.55 & 268.05 & 0.0222 & $0.0171 \pm 0.0010$ \\
$90^{\circ}$ & 223.57 & 226.58 & 225.24 & 0.0269 & $0.0212 \pm 0.0013$ \\
\hline
\end{tabular}

\section{CONCLUSION}

The calorimetric technique in conjunction with an electrically heated surface to simulate beam heating proved yet again to be an invaluable tool to investigate aspects of forced convection cooling relevant to beam-window assemblies. The new data confirm our previous conclusion that the duct equations, such as the Dittus-Boelter, Sieder-Tate and Petukhov-Kirillov equations, are not particularly suited to make estimates of the convective heat-transfer coefficient in this particular application. The fact that air was used as the coolant is not expected to affect the general validity of this statement as the underlying reason is a geometry dependent effect, namely heat transfer in the thermal entrance region of the flow. The formalism of Chang et al. for single-phase flow in a jet is seen to provide a definitive improvement in this region.

It needs to be stressed that the experimental set-up of this particular study was not primarily designed to be a close approximation to a real beam-window assembly. The main objective was to study the dependence on the jet direction in an uncomplicated assembly - one that is relatively easy to model. Modelling using a computational fluid dynamics (CFD) code, such as the program Autodesk Simulation CFD 2014, shows much promise. Predictions using the SST K-omega turbulence model are in better agreement with the measurements than those obtained with the default Kepsilon turbulence model. A weak dependence on the jet angle has been obtained from the CFD simulations, which has also been observed experimentally. This provides an explanation why Chang's formalism, for perpendicular jets, also gave a quantitatively good result for a window assembly with bulk flow parallel to the heated surfaces.

The present study only considered single-jet cooling. Multi-jet cooling, however, can be employed to enhance turbulence, hence the convective heat-transfer coefficient. Care must be taken as multi-jet configurations can also lead to the opposite effect (i.e. reducing turbulence and/or "laminarizing" the flow). It will be interesting to extend the investigation to multi-jet configurations. CFD modelling may be very powerful here, guiding the design towards turbulence enhancement and avoiding turbulence reduction. 


\section{ACKNOWLEDGMENTS}

This work was financially supported by the National Research Foundation (NRF Grant no. 85507).

\section{REFERENCES}

[1] D. Hirdes, E. Georg, H. Wollnik and R. Brandt, "Metal foils as windows of a gas-jet thermalization chamber at a heavy-ion beam." Nucl. Instr. and Meth. 130, 15-18 (1975).

[2] C. Crouzel, C. Le Poec, E. Jarry, R. Knipper and D. Comar, "Automated radioisotope production devices adapted to a medical cyclotron." Nucl. Instr. and Meth. 165, 341-344 (1979).

[3] J.H. Zaidi, S.M. Qaim and G. Stöcklin, "Excitation functions of deuteron induced nuclear reactions on natural tellurium and enriched ${ }^{122} \mathrm{Te}$ : Production of ${ }^{123} \mathrm{I}$ via the ${ }^{122} \mathrm{Te}(\mathrm{d}, \mathrm{n}){ }^{123} \mathrm{I}$-process." Appl. Radiat. Isot. 34, 1425 1430 (1983).

[4] V.S. Shaiju, S.D. Sharma, R. Kumar and B. Sarin, "Target foil rupture scenario and provision for handling different models of medical cyclotrons used in India." J. Med. Phys. 34, 161-166 (2009).

[5] J.O. Liljenzin, The temperature of thin foils in ion beams. Lawrence Berkeley Laboratory Report LBL-1912, Berkeley, California, USA, 1973.

[6] F.W. Dittus and L.M.K. Boelter, "Heat transfer in automobile radiators of the tubular type." Pub. Eng. 2, 443-461 (1930). Reprinted in Int. Comm. Heat Mass Transfer 12, 3-22 (1985).

[7] G.F. Steyn, F.M. Nortier and S.J. Mills, "Helium cooling of a double-foil window for external cyclotron beams." Nucl. Instr. and Meth. A 292, 35-44 (1990).

[8] G.F. Steyn and C.J. Stevens, "The importance of thermal entrance effects in gas-cooled beam windows for radioisotope production targets." Nucl. Instr. and Meth. A 373, 10-17 (1996).

[9] W.M. Kays and H.C. Perkins, "Forced convection, internal flows in ducts." In: W.M. Rohsenhow, J.P. Hartnett, E.N. Ganić (Eds.), Handbook of Heat Transfer Fundamentals, $2^{\text {nd }}$ Edition, McGraw Hill, 1985, pp. 7-1 $-7-23$.

[10] C.T. Chang, G. Kojasoy, F. Landis and S. Downing, "Confined single- and multiple-jet impingement heat transfer - I. Turbulent submerged liquid jets." Int. J. Heat Mass Transfer 38, 833-842 (1995).

[11] K. Gagnon, J.S. Wilson and S.A. McQuarrie, "Thermal modelling of a solid cyclotron target using finite element analysis: An experimental validation." In: Proc. $13^{\text {th }}$ Int. Workshop on Targetry and Target Chemistry (WTTC 13), Roskilde, Denmark, 2010, paper 011. Available from <http://wttc.triumf.ca $>$.

[12] O. Kwon and F.E. Ames, Advanced k-epsilon Modeling of Heat Transfer. NASA Contractor Report 4679, NASA Scientific and Technical Information Program, 1995.

[13] J.E. Bardina, P.G. Huang and T.J. Coakley, Turbulence Modeling Validation, Testing, and Development. NASA Technical Memorandum 110446, 1997.

[14] A. Pattamatta and G. Singh, "Assessment of turbulence models in the prediction of flow field and thermal characteristics of wall jet." Frontiers in Heat and Mass Transfer 3: 023005-1 - 023005-11 (2012).

[15] J.P. Holman, Heat Transfer, 10 ${ }^{\text {th }}$ Edition. McGraw Hill, 2010.

[16] G.F. Steyn, C. Vermeulen, A.H. Botha, J.L. Conradie, J.P.A. Crafford, J.L.G. Delsink, J. Dietrich, H. du Plessis, D.T. Fourie, Z. Kormány, P.F. Rohwer, N.P. Stodart and J.G. de Villiers, "A vertical-beam target station and high-power targetry for the cyclotron production of radionuclides with medium energy protons." Nucl. Instr. and Meth. A 727, 131-144 (2013). 